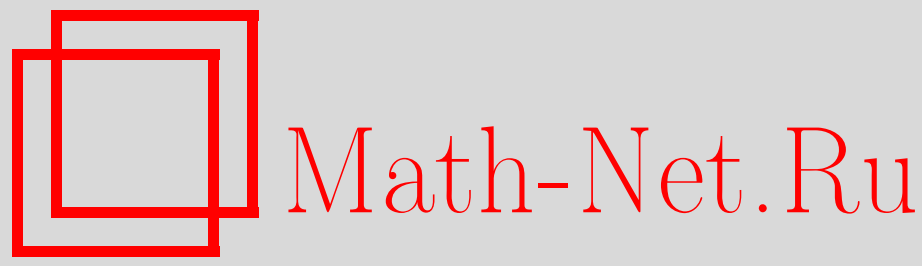

Э. Л. Аэро, А. Н. Булыгин, Ю. В. Павлов, Решения уравнения синус-Гордон с переменной амплитудой, ТМФ, 2015, том 184, номер 1, 79-91

DOI: https://doi.org/10.4213/tmf8821

Использование Общероссийского математического портала Math-Net.Ru подразумевает, что вы прочитали и согласны с пользовательским соглашением http://www . mathnet.ru/rus/agreement

Параметры загрузки:

IP : 3.93 .64 .190

26 апреля 2023 г., 14:34:31

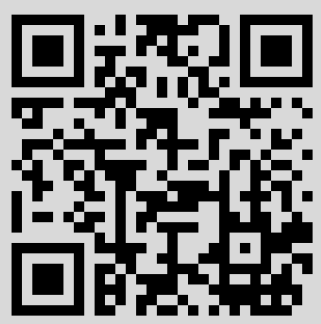




\title{
ФИЗИКА
}

Том 184, № 1

июль, 2015

(C) 2015 г. $\quad$ Э. Л. Аэро*, А. Н. Булыгин*, Ю. В. Павлов*

\section{РЕШЕНИЯ УРАВНЕНИЯ СИНУС-ГОРДОН С ПЕРЕМЕННОЙ АМПЛИТУДОЙ}

\begin{abstract}
Излагаются способы построения функционально-инвариантных решений $u(x, y, z, t)$ уравнения синус-Гордон с переменной амплитудой в $3+1$ измерениях. Решения $u(x, y, z, t)$ найдены в виде произвольной функции, которая зависит от одной $(\alpha(x, y, z, t))$ или двух $(\alpha(x, y, z, t), \beta(x, y, z, t))$ специально построенных функций. Решения $f(\alpha)$ и $f(\alpha, \beta)$ относятся к классу функционально-инвариантных, а функции $\alpha(x, y, z, t), \beta(x, y, z, t)$ называются анзацами. Анзацы $(\alpha, \beta)$ определяются как корни алгебраических или смешанных (алгебраических и дифференциальных в частных производных первого порядка) уравнений. Уравнения, определяющие анзацы, также содержат произвольные функции, зависящие от $(\alpha, \beta)$. Предложенные способы позволяют найти $u(x, y, z, t)$ для частного, но широкого класса амплитуд, как регулярных, так и сингулярных, и легко обобщаются на случай пространства любого числа измерений.
\end{abstract}

Ключевые слова: уравнение синус-Гордон, волновое уравнение, уравнение эйконала, функционально-инвариантные решения, анзац.

DOI: $10.4213 / \mathrm{tmf} 8821$

\section{1. ВВЕДЕНИЕ}

Уравнение синус-Гордон (СГ)

$$
u_{x x}+u_{y y}+u_{z z}-\frac{u_{t t}}{v^{2}}=p_{0} \sin u,
$$

где $p_{0}=$ const, a нижний индекс обозначает частную производную по соответствующей переменной, появляется во многих разделах современного естествознания. Уравнение (1) описывает движение дислокаций в твердых телах [1], деформацию нелинейной кристаллической решетки [2], распространение флюксонов в джозефсоновских линиях передач [3], ориентацию спинов в ферромагнетиках [4], ориентационную структуру жидких кристаллов [5], распространение резонансных ультракоротких оптических импульсов [6], фазовые переходы "соразмерность-несоразмерность" [7]. Оно также возникает при моделировании процессов, протекающих в земной коре [8], при описании метрики поверхностей [9], в молекулярной биологии [10], моделях теории поля и физике элементарных частиц [11], [12].

* Институт проблем машиноведения РАН, Санкт-Петербург, Россия. E-mail: bulygin_an@mail.ru 
K настоящему времени разработаны эффективные методы решения уравнения СГ с постоянной амплитудой и практически отсутствуют методы решения уравнения СГ с переменной амплитудой. Это существенно ограничивает область применения уравнения СГ, так как многие физические явления и технологические процессы описываются уравнением СГ с переменной амплитудой

$$
u_{x x}+u_{y y}+u_{z z}-\frac{u_{t t}}{v^{2}}=p(x, y, z, t) \sin u .
$$

Так, в механике нелинейной кристаллической решетки случай постоянной амплитуды описывает деформацию идеальной кристаллической решетки полем однородных напряжений. Деформация реальной решетки с дефектами структуры (дислокации, дисклинации, зародыши пор, трещины и т. д.) даже однородными напряжениями, а тем более полем неоднородных напряжений описывается уравнением СГ с переменной амплитудой. В механике жидких кристаллов случай $p=$ const моделирует деформацию длинных осей электромагнитным полем при условии, что ориентационный континуум не содержит дефектов, а электромагнитное поле однородно. В противном случае $p(x, y, z, t) \neq$ const. В теории поверхностей уравнение СГ с $p(x, y, z, t)=$ const описывает метрику сетей Чебышева на поверхности с постоянной кривизной. Если кривизна поверхности изменяется, то $p(x, y, z, t) \neq$ const. При моделировании распространения волн в земной коре [13] уравнение СГ с постоянной амплитудой описывает случай, когда механические свойства земной коры являются однородными, что, несомненно, является большой идеализацией. Из приведенных примеров ясно, что область применения уравнения СГ существенно расширяется, если будут найдены решения уравнения СГ с переменной амплитудой.

При описании реальных сред дефекты структуры принято моделировать функциями, имеющими соответствующие сингулярности (точка, линия, поверхность и т. д.) Сингулярности могут быть стационарными и подвижными, т. е. распространяться с некоторой скоростью. Они могут различаться мерой роста (логарифмическая, степенная и т. д.). Широко распространены среды, у которых неоднородности описываются регулярными функциями, не имеющими сингулярностей, причем нередко неоднородности имеют правильное геометрическое расположение в пространстве. Из сказанного ясно, что математическая модель, построенная на основе уравнения $\mathrm{CГ}$, будет адекватной, если амплитуда $p(x, y, z, t)$ будет учитывать особенности строения реальных сред.

Ниже излагается метод решения уравнения СГ с переменной амплитудой. Метод основан на следующих утверждениях, которые доказываются прямыми расчетами.

УтвЕРЖДЕНИЕ 1. Если функиия $\varphi(x, y, z, t)$ одновременно удовлетворяет уравнениям

$$
\begin{gathered}
\varphi_{x x}+\varphi_{y y}+\varphi_{z z}-\frac{\varphi_{t t}}{v^{2}}=0, \\
\varphi_{x}^{2}+\varphi_{y}^{2}+\varphi_{z}^{2}-\frac{\varphi_{t}^{2}}{v^{2}}=p(x, y, z, t),
\end{gathered}
$$

mo

$$
u=4 \operatorname{arctg} e^{\varphi(x, y, z, t)}
$$

есть решение уравнения (2). 
ДокАзАтЕльство. Действительно, если подставить (4) в (2) и учесть, что

$$
\sin \left(4 \operatorname{arctg} e^{\varphi}\right)=\frac{4 e^{\varphi}}{1+e^{2 \varphi}}-\frac{8 e^{3 \varphi}}{\left(1+e^{2 \varphi}\right)^{2}},
$$

то уравнение (2) примет вид

$$
\varphi_{x x}+\varphi_{y y}+\varphi_{z z}-\frac{\varphi_{t t}}{v^{2}}=\left(\varphi_{x}^{2}+\varphi_{y}^{2}+\varphi_{z}^{2}-\frac{\varphi_{t}^{2}}{v^{2}}-p(x, y, z, t)\right) \operatorname{th} \varphi
$$

Из (6) следует справедливость утверждения 1.

УтВЕРЖДЕНИЕ 2. Если функиия $\varphi(x, y, z, t)$ одновременно удовлетворяет уравнениям

$$
\begin{aligned}
\varphi_{x x}+\varphi_{y y}+\varphi_{z z}-\frac{\varphi_{t t}}{v^{2}} & =p(x, y, z, t), \\
\varphi_{x}^{2}+\varphi_{y}^{2}+\varphi_{z}^{2}-\frac{\varphi_{t}^{2}}{v^{2}} & =0
\end{aligned}
$$

mo

$$
u=2 \operatorname{arctg} e^{\varphi(x, y, z, t)}
$$

есть решение уравнения (2).

ДокАЗАТЕльство. Утверждение доказывается аналогично утверждению 1.

Если подставить (8) в (2) и учесть, что

$$
\sin \left(2 \operatorname{arctg} e^{\varphi}\right)=\frac{2 e^{\varphi}}{1+e^{2 \varphi}}
$$

то уравнение (2) примет вид

$$
\varphi_{x x}+\varphi_{y y}+\varphi_{z z}-\frac{\varphi_{t t}}{v^{2}}-p(x, y, z, t)=\left(\varphi_{x}^{2}+\varphi_{y}^{2}+\varphi_{z}^{2}-\frac{\varphi_{t}^{2}}{v^{2}}\right) \operatorname{th} \varphi
$$

Из (10) очевидна справедливость утверждения 2.

Полученные результаты сводят решение уравнения (2) к нахождению функции $\varphi(x, y, z, t)$, которая одновременно удовлетворяет системе уравнений (3) или (7). Функцию $\varphi(x, y, z, t)$ можно найти, если применить методы построения функционально-инвариантных решений дифференциальных уравнений [14]-[25].

\section{2. ФУНКЦИОНАЛЬНО-ИНВАРИАНТНЫЕ РЕШЕНИЯ УРАВНЕНИЯ СГ С ПЕРЕМЕННОЙ АМПЛИТУДОЙ}

Решение дифференциального уравнения называется функционально-инвариантным, если оно имеет вид произвольной функции, зависящей от анзаца. Анзац является решением одного или нескольких уравнений. Уравнения могут быть алгебраическими, дифференциальными или смешанного вида. Существуют функционально-инвариантные решения, зависящие от двух и более анзацев.

Идею о существовании функционально-инвариантных решений высказал Якоби. Для уравнения Лапласа он отметил, что аргумент произвольной функции, входящей 
в решение дифференциального уравнения в частных производных, должен удовлетворять и уравнению характеристик [26]. Форсайт [15] нашел функционально-инвариантные решения уравнений Лапласа, волнового уравнения в пространстве любого числа измерений и уравнения Гельмгольца в трехмерном пространстве. Фундаментально и последовательно идею Якоби применительно к волновому уравнению разработал Бейтмен [14] при исследовании электромагнитных волн. Соболев и Смирнов [16]-[19] успешно применяли метод построения функционально-инвариантных решений волнового уравнения для решения задач дифракции и распространения звуковых волн в твердых однородных и слоистых средах. В разработку теории метода большой вклад сделал Еругин [20]. Функционально-инвариантные решения уравнения СГ с постоянной амплитудой нашли авторы [22].

2.1. Решения с одним анзацем. При построении функционально-инвариантных решений дифференциальных уравнений с частными производными основным является вопрос нахождения анзаца. Как правило, он выбирается из решений специальных уравнений.

Пусть анзац $\tau$ есть корень алгебраического уравнения

$$
x \xi(\tau)+y \eta(\tau)+z \zeta(\tau)-v^{2} t \tau=\frac{s^{2}+q^{2}}{2},
$$

где

$$
s^{2}=x^{2}+y^{2}+z^{2}-v^{2} t^{2}, \quad q^{2}=\xi^{2}(\tau)+\eta^{2}(\tau)+\zeta^{2}(\tau)-v^{2} \tau^{2},
$$

а $\xi(\tau), \eta(\tau), \zeta(\tau)$ - произвольные функции $\tau$. Уравнение (11) неявным образом определяет зависимость $\tau$ от времени и пространственных координат. Из него по правилу дифференцирования неявных функций находим частные производные анзаца $\tau(x, y, z, t)$ и, учитывая соотношения

$$
\xi_{\tau} \tau_{x}+\eta_{\tau} \tau_{y}+\zeta_{\tau} \tau_{z}+\tau_{t}=1, \quad \nu_{x} \tau_{x}+\nu_{y} \tau_{y}+\nu_{z} \tau_{z}-\frac{\nu_{t} \tau_{t}}{v^{2}}=1
$$

где

$$
\nu=\xi_{\tau}(x-\xi)+\eta_{\tau}(y-\eta)+\zeta_{\tau}(z-\zeta)-v^{2}(t-\tau),
$$

находим, что анзац $\tau$ удовлетворяет уравнениям

$$
\begin{gathered}
\tau_{x}^{2}+\tau_{y}^{2}+\tau_{z}^{2}-\frac{\tau_{t}^{2}}{v^{2}}=0 \\
\tau_{x x}+\tau_{y y}+\tau_{z z}-\frac{\tau_{t t}}{v^{2}}=p(x, y, z, t), \quad p(x, y, z, t)=\frac{2}{\nu} .
\end{gathered}
$$

На основании (15) и утверждения 2 произвольная функция, зависящая от $\tau$, $\varphi(x, y, z, t)=f(\tau)$, удовлетворяет уравнениям (7) и, следовательно, функция

$$
u=2 \operatorname{arctg} e^{f(\tau)}
$$

является решением уравнения СГ (2) с амплитудой

$$
p(x, y, z, t)=\frac{2}{\nu} f_{\tau} .
$$



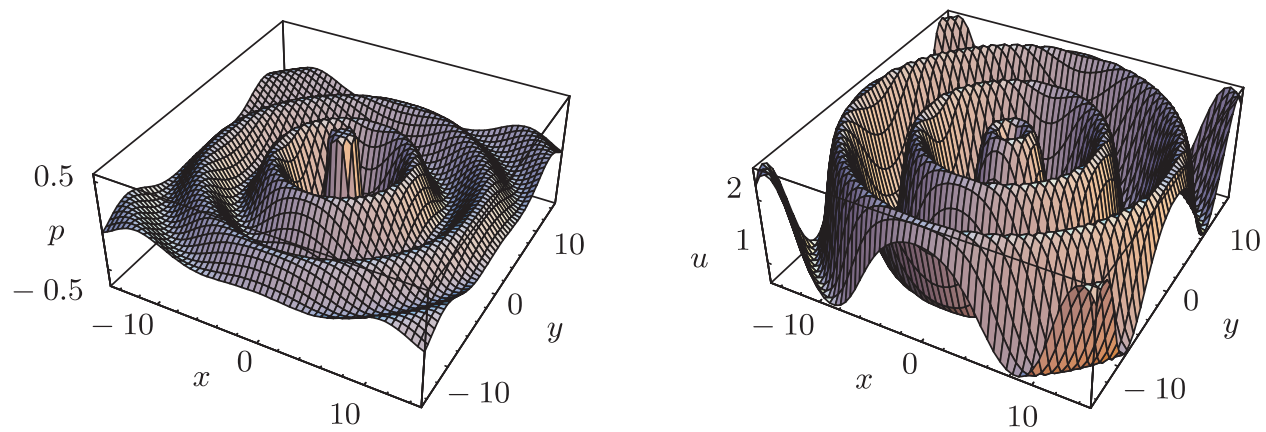

a
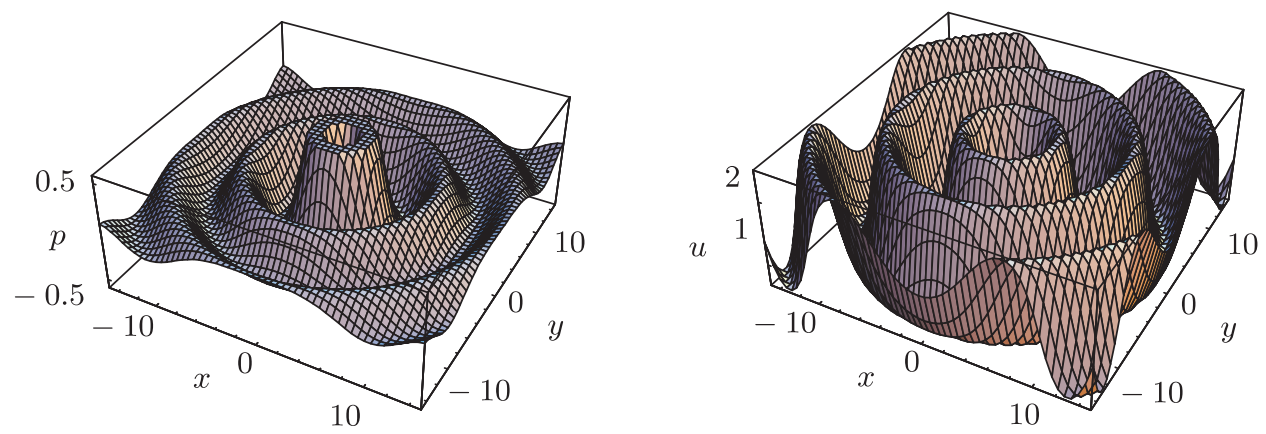

Рис. 1. Амплитуда $p$ и решение $u(x, y, 0, t)$, соответствующее $(21)$, при $t=0$ (а) и $t=10$ (б).

Функция $\varphi=f(\tau) / \nu$ удовлетворяет уравнениям (3), если амплитуда имеет вид

$$
p(x, y, z, t)=\frac{f^{2}}{\nu^{4}}\left(2 W+\xi_{\tau}^{2}+\eta_{\tau}^{2}+\zeta_{\tau}^{2}-v^{2}\right)-2 \frac{f f_{\tau}}{\nu^{3}}
$$

и

$$
W=\xi_{\tau \tau}(x-\xi)+\eta_{\tau \tau}(y-\eta)+\zeta_{\tau \tau}(z-\zeta)-\xi_{\tau}^{2}-\eta_{\tau}^{2}-\zeta_{\tau}^{2}+v^{2}
$$

При доказательстве этого утверждения необходимо наряду с (13)-(15) учесть уравнения, которым удовлетворяет функция $\nu$ :

$$
\begin{gathered}
\nu_{x}^{2}+\nu_{y}^{2}+\nu_{z}^{2}-\frac{\nu_{t}^{2}}{v^{2}}=2 W+\xi_{\tau}^{2}+\eta_{\tau}^{2}+\zeta_{\tau}^{2}-v^{2}, \\
\nu_{x x}+\nu_{y y}+\nu_{z z}-\frac{\nu_{t t}}{v^{2}}=\frac{2}{\nu}\left[2 W+\xi_{\tau}^{2}+\eta_{\tau}^{2}+\zeta_{\tau}^{2}-v^{2}\right] .
\end{gathered}
$$

На основании утверждения 1 функция

$$
u=4 \operatorname{arctg} e^{f(\tau) / \nu}
$$

является решением уравнения СГ с амплитудой (18). Полученные решения позволяют находить большое число частных решений путем конкретизации произвольных 
функций. Отметим два элементарных решения:

1) $\quad \xi=0, \quad \eta=0, \quad \zeta=0$,

$$
\tau=t \mp \frac{R}{v}, \quad \nu=\mp v R, \quad R=\sqrt{x^{2}+y^{2}+z^{2}},
$$

2) $\quad \xi=x_{1} \tau, \quad \eta=x_{2} \tau, \quad \zeta=x_{3} \tau, \quad x_{1}^{2}+x_{2}^{2}+x_{3}^{2}=v^{2}$,

$$
\tau=\frac{s^{2}}{2 \nu}, \quad \nu=x x_{1}+y x_{2}+z x_{3}-v^{2} t, \quad s^{2}=x^{2}+y^{2}+z^{2}-v^{2} t^{2}
$$

На рис. 1 приведены графики амплитуды и решения (21) уравнения СГ для разных моментов времени при $v=1, z=0$ и $f(\tau)=\sin \tau$. Решение $u(x, y, 0, t)$ имеет вид стоячей волны.

Функционально-инвариантные решения уравнения СГ можно построить, если анзац $\tau(x, y, z, t)$ выбрать из решений системы уравнений

$$
\begin{gathered}
x \xi(\alpha, \beta, \tau)+y \eta(\alpha, \beta, \tau)+z \zeta(\alpha, \beta, \tau)-v^{2} t \theta(\alpha, \beta, \tau)=\frac{s^{2}+q^{2}}{2}, \\
x \xi_{\alpha}+y \eta_{\alpha}+z \zeta_{\alpha}-v^{2} t \theta_{\alpha}=\frac{\left(q^{2}\right)_{\alpha}}{2} \\
x \xi_{\beta}+y \eta_{\beta}+z \zeta_{\beta}-v^{2} t \theta_{\beta}=\frac{\left(q^{2}\right)_{\beta}}{2},
\end{gathered}
$$

где $q^{2}=\xi^{2}+\eta^{2}+\zeta^{2}-v^{2} \theta^{2}$.

Уравнение (23) алгебраическое. По форме оно совпадает с уравнением (11), но отличается от него тем, что функции $\xi, \nu, \zeta, \theta$ зависят не от одного аргумента $\tau$, а от трех аргументов $\alpha, \beta, \tau$. Уравнения (24), (25) - дифференциальные уравнения в частных производных первого порядка. Из (23)-(25) можно найти частные производные анзаца $\tau$ и доказать, что он удовлетворяет уравнениям $(7)$ с функцией

$$
p(x, y, z, t)=\frac{1}{\nu}\left[3-\left(\xi_{x}+\eta_{y}+\zeta_{z}+\theta_{t}\right)\right] .
$$

Следовательно, функция $u=2 \operatorname{arctg} e^{f(\tau)}$ является решением уравнения СГ (2) с амплитудой (26).

Из приведенного решения получаются интересные элементарные решения:

1) $\quad \xi=v \tau \cos \alpha \cos \beta, \quad \eta=v \tau \cos \alpha \sin \beta, \quad \zeta=v \tau \sin \alpha, \quad \theta=\tau$,

$$
\tau=\frac{1}{2}\left(t+\frac{R}{v}\right), \quad p(x, y, z, t)=\frac{f_{\tau}}{v R}
$$

2) $\quad \xi=v \tau, \quad \eta=\zeta=0 \tau, \quad \theta=\tau$,

$$
\tau=\frac{s^{2}}{2 v(x-v t)}, \quad p(x, y, z, t)=\frac{2}{v(x-v t)} f_{\tau} \text {; }
$$


3) $\quad \xi=\tau \cos \alpha, \quad \eta=\tau \sin \alpha, \quad \zeta=\tau \operatorname{sh} \beta, \quad \theta=\frac{\tau}{v} \operatorname{ch} \beta$,

$\tau=\frac{1}{2}\left[\sqrt{x^{2}+y^{2}}+\sqrt{v^{2} t^{2}-z^{2}}\right], \quad p(x, y, z, t)=\frac{1}{2}\left[\frac{1}{\sqrt{x^{2}+y^{2}}}-\frac{1}{\sqrt{v^{2} t^{2}-z^{2}}}\right] f_{\tau} ;$

4) $\xi=\tau \cos \alpha \operatorname{sh} \beta, \quad \eta=\tau \sin \alpha \operatorname{sh} \beta, \quad \zeta=\tau, \quad \theta=\frac{\tau}{v} \operatorname{ch} \beta$,

$\tau=\frac{1}{2}\left[z+\sqrt{v^{2} t^{2}-x^{2}-y^{2}}\right], \quad p(x, y, z, t)=\frac{-f_{\tau}}{\sqrt{v^{2} t^{2}-x^{2}-y^{2}}}$.

На рис. 2 приведены графики амплитуды и решения (28) уравнения СГ при $v=1$, $z=0$ для случая $f(\tau)=\tau$. Решение $u(x, y, 0, t)$ представляет собой суперпозицию двух типов возмущений. Первое подобно кинку. Плоскость разрыва движется со скоростью $v$. Второе подобно солитону. Поперечные размеры второго решения уве-
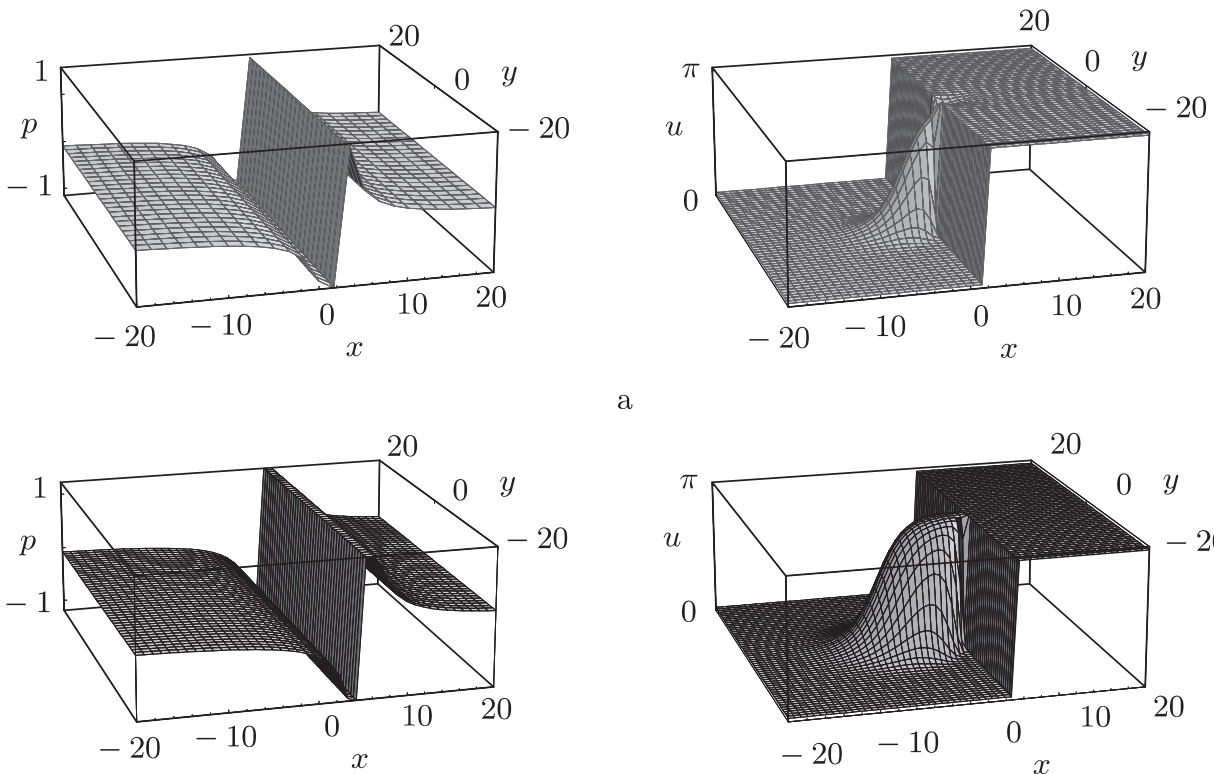

a
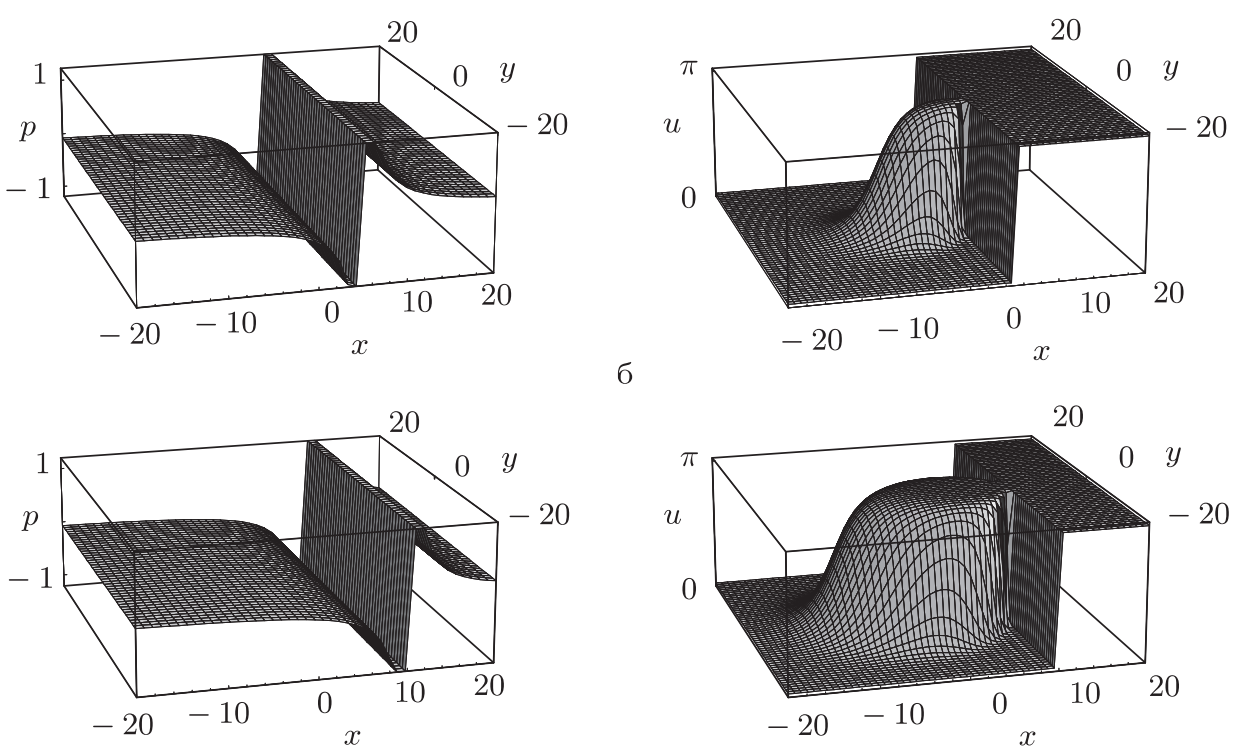

B

Рис. 2. Амплитуда $p$ и решение $u(x, y, 0, t)$, соответствующее $(28)$, при $t=2(\mathrm{a}), t=5$ (б) и $t=10$ (в). 
личиваются со скоростью $v$, а высота достигает своего наибольшего значения $u=\pi$, со временем вершина второго решения приближается к плоскости.

2.2. Решения с двумя анзацами. Существуют функционально-инвариантные решения уравнения (2) с двумя анзацами. Пусть анзац $\alpha(x, y, z, t)$ есть корень уравнения

$$
x l(\alpha)+y m(\alpha)+z n(\alpha)-v^{2} t w(\alpha)+g(\alpha)=0,
$$

а анзац $\beta(x, y, z, t)$ имеет вид

$$
\beta(x, y, z, t)=x l_{\alpha}+y m_{\alpha}+z n_{\alpha}-v^{2} t w_{\alpha}+g_{\alpha} .
$$

Здесь $l(\alpha), m(\alpha), n(\alpha), w(\alpha), g(\alpha)$ - произвольные функции $\alpha$, связанные условием

$$
l^{2}+m^{2}+n^{2}=v^{2} w^{2} .
$$

Из (31), (32) можно найти частные производные анзацев $(\alpha, \beta)$ и убедиться, что произвольная функция от анзацев $(\alpha, \beta)$

$$
\varphi=F(\alpha, \beta)
$$

удовлетворяет системе уравнений

$$
\begin{aligned}
\varphi_{x}^{2}+\varphi_{y}^{2}+\varphi_{z}^{2}-\frac{\varphi_{t}^{2}}{v^{2}} & =F_{\beta}^{2} C^{2}(\alpha), \\
\varphi_{x x}+\varphi_{y y}+\varphi_{z z}-\frac{\varphi_{t t}}{v^{2}} & =\left(F_{\beta \beta}+\frac{2}{\beta} F_{\beta}\right) C^{2}(\alpha),
\end{aligned}
$$

где

$$
C^{2}(\alpha)=l_{\alpha}^{2}+m_{\alpha}^{2}+n_{\alpha}^{2}-v^{2} w_{\alpha}^{2} .
$$

Функция (34) будет решением однородного волнового уравнения, если

$$
F=A(\alpha)+\frac{B(\alpha)}{\beta},
$$

где $A(\alpha), B(\alpha)$ - произвольные функции анзаца $\alpha$. Подставив (37) в (35), получим

$$
\varphi_{x}^{2}+\varphi_{y}^{2}+\varphi_{z}^{2}-\frac{\varphi_{t}^{2}}{v^{2}}=\frac{B^{2}(\alpha) C^{2}(\alpha)}{\beta^{4}}
$$

Таким образом, функция

$$
u=4 \operatorname{arctg}\left[e^{A(\alpha)+B(\alpha) / \beta}\right]
$$

есть решение уравнения СГ (2) с амплитудой

$$
p(x, y, z, t)=\frac{B^{2}(\alpha) C^{2}(\beta)}{\beta^{4}} .
$$

Приведем простой пример данного решения. Примем, что $l=\cos \alpha, m=\sin \alpha$, $n=0, v w=1, g=0$. Тогда анзац имеет вид

$$
\begin{aligned}
\alpha & =-\gamma+(-1)^{k} \arcsin \frac{v t}{\rho}, \quad k=0, \pm 1, \ldots, \\
\operatorname{tg} \gamma & =\frac{x}{y}, \quad \rho=\sqrt{x^{2}+y^{2}}, \quad \beta^{2}=\rho^{2}-v^{2} t^{2},
\end{aligned}
$$


амплитуда

$$
p(x, y, z, t)=\frac{B^{2}(\alpha)}{\beta^{4}}
$$

Решения уравнений (7) можно получить, если в качестве анзацев $(\alpha, \beta)$ взять корни уравнений

$$
\begin{aligned}
x \xi(\alpha, \beta)+y \eta(\alpha, \beta)+z \zeta(\alpha, \beta)-v^{2} t \tau(\alpha, \beta) & =\frac{s^{2}+q^{2}}{2}, \\
x l(\alpha, \beta)+y m(\alpha, \beta)+z n(\alpha, \beta)-v^{2} t w(\alpha, \beta) & =g(\alpha, \beta) .
\end{aligned}
$$

Здесь

$$
g(\alpha, \beta)=l \xi+m \eta+n \zeta-v^{2} w \tau,
$$

а произвольные функции $l, m, n, w$ аргументов $(\alpha, \beta)$ связаны условиями

$$
l^{2}+m^{2}+n^{2}=v^{2} w^{2}, \quad l \xi_{\beta}+m \eta_{\beta}+n \zeta_{\beta}=v^{2} w \tau_{\beta} .
$$

Величины $\left(s^{2}, q^{2}\right)$ определены в $(12)$.

Из уравнений (43) находим частные производные анзацев $(\alpha, \beta)$ и убеждаемся, что производные первого порядка удовлетворяют однородным уравнениям

$$
\begin{array}{r}
\alpha_{x}^{2}+\alpha_{y}^{2}+\alpha_{z}^{2}-\frac{\alpha_{t}^{2}}{v^{2}}=0, \\
\beta_{x}^{2}+\beta_{y}^{2}+\beta_{z}^{2}-\frac{\beta_{t}^{2}}{v^{2}}=0, \\
\alpha_{x} \beta_{x}+\alpha_{y} \beta_{y}+\alpha_{z} \beta_{z}-\frac{\alpha_{t} \beta_{t}}{v^{2}}=0,
\end{array}
$$

а производные второго порядка - неоднородным уравнениям

$$
\begin{aligned}
\alpha_{x x}+\alpha_{y y}+\alpha_{z z}-\frac{\alpha_{t t}}{v^{2}} & =\frac{2}{\Delta} S, \\
\beta_{x x}+\beta_{y y}+\beta_{z z}-\frac{\beta_{t t}}{v^{2}} & =\frac{2}{\Delta}(\lambda-R),
\end{aligned}
$$

где

$$
\begin{aligned}
\Delta & =P S-R Q \\
P & =\xi_{\alpha}(x-\xi)+\eta_{\alpha}(y-\eta)+\zeta_{\alpha}(z-\zeta)-v^{2} \tau_{\alpha}(t-\tau), \\
Q & =\xi_{\beta}(x-\xi)+\eta_{\beta}(y-\eta)+\zeta_{\beta}(z-\zeta)-v^{2} \tau_{\beta}(t-\tau), \\
S & =-l_{\beta}(x-\xi)-m_{\beta}(y-\eta)-n_{\beta}(z-\zeta)+v^{2} w_{\beta}(t-\tau), \\
R & =-l_{\alpha}(x-\xi)-m_{\alpha}(y-\eta)-n_{\alpha}(z-\zeta)+v^{2} w_{\alpha}(t-\tau)+\lambda, \\
\lambda & =\xi_{\alpha} l+\eta_{\alpha} m+\zeta_{\alpha} n-v^{2} w \tau_{\alpha} .
\end{aligned}
$$

Полученные результаты позволяют утверждать, что функция $\varphi$ вида (34) удовлетворяет уравнениям (3), если

$$
p(x, y, z, t)=\frac{2 N}{\Delta}
$$

где

$$
N=\frac{\partial(l, f)}{\partial(\alpha, \beta)}(x-\xi)+\frac{\partial(m, f)}{\partial(\alpha, \beta)}(y-\eta)+\frac{\partial(n, f)}{\partial(\alpha, \beta)}(z-\zeta)-v^{2} \frac{\partial(w, f)}{\partial(\alpha, \beta)}(t-\tau) .
$$


Здесь использовано обозначение определителя Якоби

$$
\frac{\partial(\varphi, \psi)}{\partial(\alpha, \beta)}=\frac{\partial \varphi}{\partial \alpha} \frac{\partial \psi}{\partial \beta}-\frac{\partial \varphi}{\partial \beta} \frac{\partial \psi}{\partial \alpha}
$$

Следовательно, функция

$$
u=2 \operatorname{arctg}\left[e^{F(\alpha, \beta)}\right]
$$

является решением уравнения СГ (2) с амплитудой (47).

Для моделирования физических процессов и решения задач механики могут представлять интерес простейшие анзацы:

$$
\begin{aligned}
& \text { 1) } \alpha=x \pm i y, \quad \beta=z \pm v t ; \\
& \text { 2) } \alpha=x+i z \sqrt{1+c^{2}}+i c v t, \quad \beta=y+c z+v t \sqrt{1+c^{2}},
\end{aligned}
$$

где $c$ - произвольная постоянная.

Если ограничиться вещественными решениями, то функция

$$
\varphi=\frac{1}{2}[F(\alpha, \beta)+\bar{F}(\bar{\alpha}, \beta)]
$$

является решением уравнений (7), если

$$
p(x, y, z, t)=F_{\alpha} \bar{F}_{\bar{\alpha}},
$$

a

$$
u=4 \operatorname{arctg}\left[e^{(F(\alpha, \beta)+\bar{F}(\bar{\alpha}, \beta)) / 2}\right] .
$$

Здесь черта над символом обозначает комплексное сопряжение.

На рис. 3 приведены графики амплитуды и решения (53) уравнения СГ при $v=1$, $z=0$ для случая $F(\alpha, \beta)=\beta / \operatorname{ch} \alpha$. Решение $u(x, y, 0, t)$ имеет вид периодических по оси $O y$ возмущений, которые со временем изменяют размеры и форму, а высота достигает наибольшего значения $u=2 \pi$, и со временем приближается к плоскости.

Найденные решения уравнения (2) даются функциональными зависимостями (4) и (8). Можно построить функционально-инвариантные решения уравнения СГ с переменной амплитудой, имеющие другую функциональную зависимость. Будем искать решения уравнения (2) в виде

$$
u=2 \arcsin \left[\sqrt{1-\nu_{0}^{2}} \frac{\operatorname{sn}\left(\varphi, \nu_{0}\right)}{\operatorname{dn}\left(\varphi, \nu_{0}\right)}\right]
$$

либо в виде

$$
u=\pi+2 \operatorname{am}\left(\varphi, \nu_{0}\right)
$$

Здесь $\operatorname{sn}\left(\varphi, \nu_{0}\right), \operatorname{dn}\left(\varphi, \nu_{0}\right)$ - эллиптические функции Якоби, $\operatorname{am}\left(\varphi, \nu_{0}\right)$ - амплитуда Якоби, $\nu_{0}=\mathrm{const}-$ модуль, а аргумент $\varphi=\varphi(x, y, z, t)$. Подставив (54) или (55) в (2), убеждаемся, что (54) и (55) суть решения уравнения СГ с амплитудой $\nu_{0}^{2} p(x, y, z, t)$, если $\varphi(x, y, z, t)$ удовлетворяет уравнениям (3). 

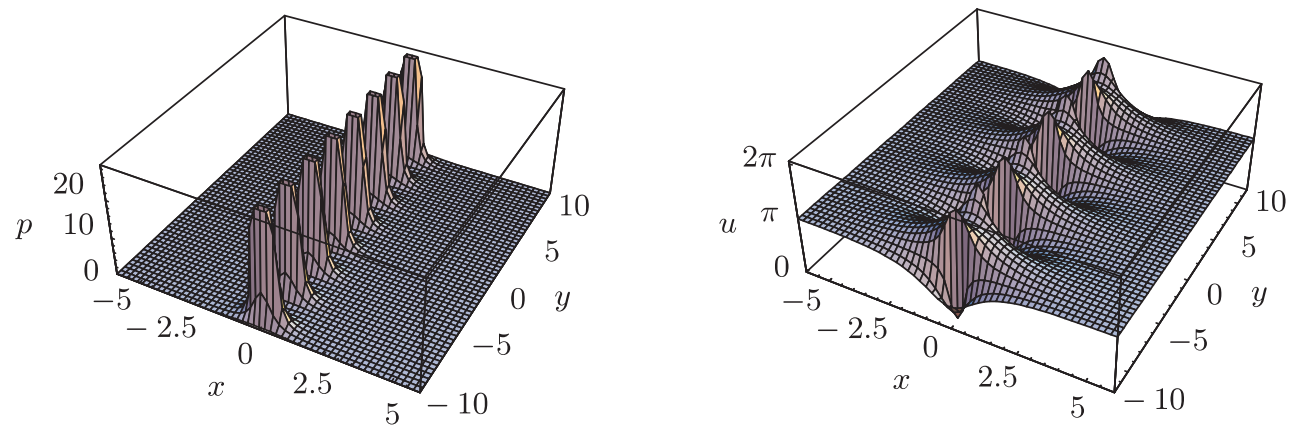

a
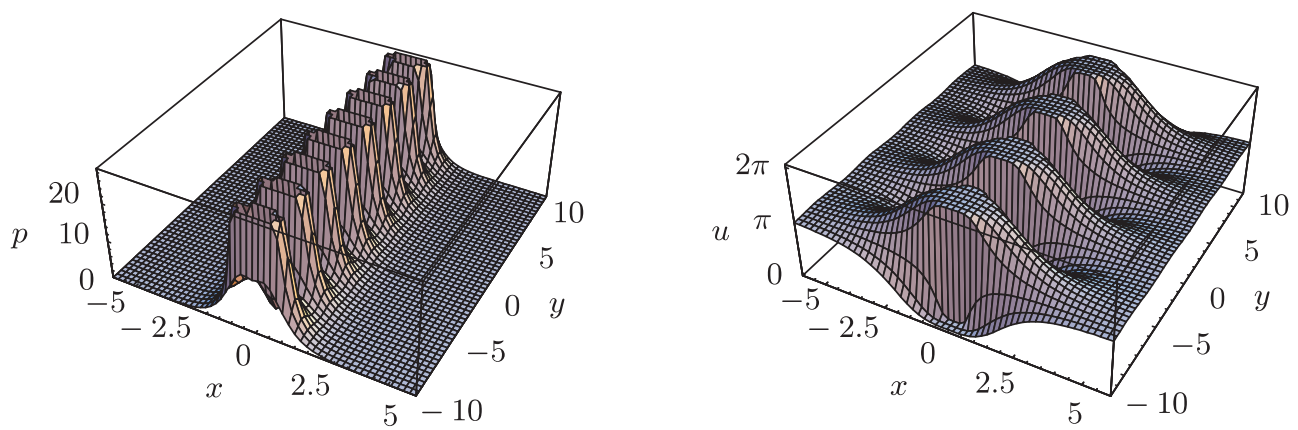

6
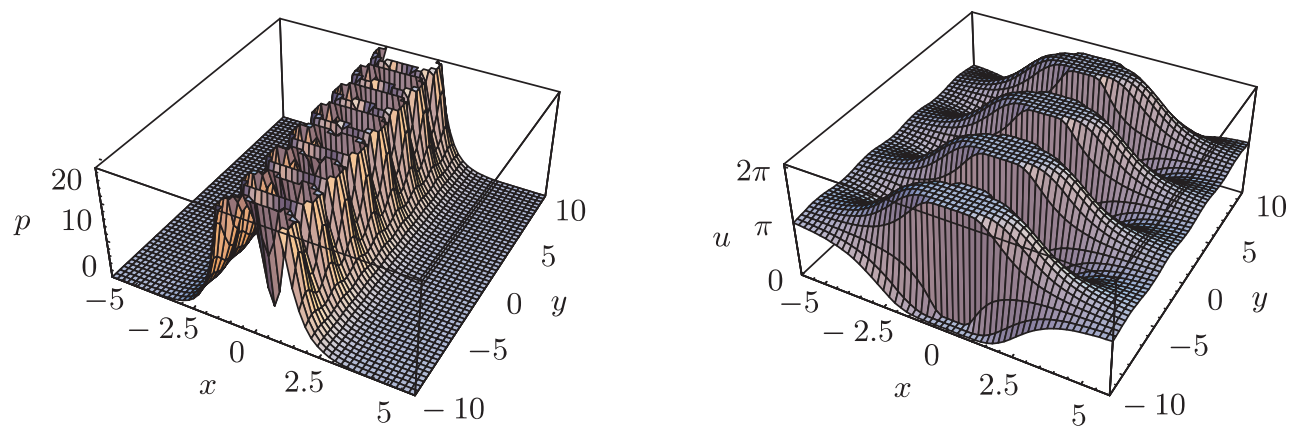

B

Рис. 3. Амплитуда $p$ и решение $u(x, y, 0, t)$, соответствующее (53), при $t=1$ (а), $t=5$ (б) и $t=10$ (в).

Отметим также, что если функция $\varphi(x, y, z, t)$ удовлетворяет уравнениям (3) или (7), то функция

$$
V=e^{\varphi(x, y, z, t)}
$$

является решением уравнения

$$
\left.\frac{\partial^{2} V}{\partial x^{2}}+\frac{\partial^{2} V}{\partial y^{2}}+\frac{\partial^{2} V}{\partial z^{2}}-\frac{1}{v^{2}} \frac{\partial^{2} V}{\partial t^{2}}=p_{(} x, y, z, t\right) V
$$


Таким образом, найденные выше решения уравнений (3) можно использовать в (54)-(56) и получить новые решения уравнения (2) в форме (54), (55) и уравнения (57).

\section{3. ЗАКЛЮЧЕНИЕ}

Предложены способы построения функционально-инвариантных решений уравнений СГ с переменной амплитудой в $3+1$ измерениях. Они легко обобщаются на случай пространства любого числа измерений. Более того, чем выше размерность пространства, тем больше существует возможных типов уравнений, корни которых могут быть анзацами функционально-инвариантных решений [20]. С ростом размерности также увеличивается число произвольных функций, входящих в уравнения для определения анзаца, что увеличивает множество решений уравнения СГ с переменной амплитудой.

Предложенные способы позволяют получить решения для амплитуд специального вида. Однако найденные решения образуют достаточно широкий класс функций. Это следует из самого метода решения и способа его построения. Решения имеют вид произвольной функции, зависящей от одного или двух анзацев. Анзацы находятся из одного уравнения или из системы уравнений, которые содержат произвольные функции. И хотя они должны удовлетворять некоторым условиям (разным для разных способов), свобода в определении анзаца (анзацев) остается.

Можно надеяться, что предложенный подход позволит адекватно описать физические процессы в средах с реальной структурой и найти решения конкретных инженерных задач, а свобода выбора анзацев будет достаточной, чтобы удовлетворить требуемым начальным и граничным условиям.

Благодарности. Работа поддержана РФФИ (грант № 13-01-00224_a).

\section{Список литературы}

[1] J. Frenkel, T. Kontorova, Acad. Sci. U.S.S.R. J. Phys., 1 (1939), 137-149.

[2] Э.Л. Аэро, А.Н. Булыгин, Изв. РАН. Сер. Механика твердого тела, 2007, №5, 170-187.

[3] P. Guéret, IEEE Trans. Magnetics, 11:2 (1975), 751-754.

[4] Р. Додд, Дж. Эйлбек, Дж. Гиббон, Х. Моррис, Солитоны и нелинейные волновые уравнения, Мир, М., 1988.

[5] П. Ж. де Жен, Физика жидких кристаллов, Мир, М., 1977.

[6] К. Лонгрен, Э. Скотт, Солитонъ в действии, Мир, М., 1981.

[7] W. L. McMillan, Phys. Rev. B, 14:4 (1976), 1496-1502.

[8] В. Г. Быков, Нелинейные волновые процессы в геологических средах, Дальнаука, Владивосток, 2000.

[9] П. Л. Чебышев, УМН, 1:2(12) (1946), 38-42.

[10] А. С. Давыдов, Солитоны в биоэнергетике, Наукова думка, Киев, 1986.

[11] Л. А. Тахтаджян, Л. Д. Фаддеев, Гамильтонов подход в теории солитонов, Наука, М., 1986.

[12] Л. А. Тахтаджян, Л. Д. Фаддеев, ТМФ, 21:2 (1974), 160-174.

[13] И. А. Гарагаш, В.Н. Николаевский, Вычисл. мех. сплошных сред, 2:4 (2009), 44-66.

[14] Г. Бейтмен, Математическая теория распространения электромагнитных волн, Наука, М., 1958. 
[15] A. R. Forsyth, Messenger Math., 27 (1898), 99-118.

[16] V. Smirnoff, S. Soboleff, "Sur une méthode nouvelle dans le problème plan des vibrations élastiques", Труды сейсмологического ин-та, № 20, Изд-во АН СССР, Л., 1932.

[17] V. Smirnoff, S. Soboleff, C. R. Acad. Sci. Paris, 194 (1932), 1437-1439.

[18] С.Л. Соболев, "Функционально-инвариантные решения волнового уравнения", Тр. Физ.-матем. ин-та им. В. А. Стеклова, 5, Изд-во АН СССР, Л., 1934, 259-264.

[19] С. Л. Соболев, Избранные труды, т. І: Уравнения математической физики. Вычислительная математика и кубатурные формулы, Изд-во ин-та математики им. С. Л. Соболева СО РАН, Новосибирск, 2003; т. ІІ: Функииональный анализ. Дифференциальные уравнения с частными производными, Изд-во ин-та математики им. С. Л. Соболева СО РАН, Новосибирск, 2006.

[20] Н. П. Еругин, Ученые зап. Ленингр. ун-та, 15 (1948), 101-134.

[21] М. М. Смирнов, Докл. АН СССР, 67 (1949), 977-980.

[22] Э. Л. Аэро, А. Н. Булыгин, Ю. В. Павлов, ТМФ, 158:3 (2009), 370-377.

[23] Э. Л. Аэро, А. Н. Булыгин, Ю. В. Павлов, Нелинейный мир, 7:7 (2009), 513-517.

[24] Э.Л. Аэро, А.Н. Булыгин, Ю.В. Павлов, Дифферени. уравнения, 47:10 (2011), $1428-1438$.

[25] E. L. Aero, A. N. Bulygin, Yu. V. Pavlov, Appl. Math. Comput., 223 (2013), 160-166.

[26] C. G. J. Jacobi, J. Reine Angew. Math., 36 (1848), 113-134; "Über eine particuläre Lösung der partiellen Differentialgleichung $\frac{\partial^{2} V}{\partial x^{2}}+\frac{\partial^{2} V}{\partial y^{2}}+\frac{\partial^{2} V}{\partial z^{2}}$ ", C. G. J. Jacobi's Gesammelte Werke, 2, ред. C. G. J. Jacobi, C. W. Borchardt, Verlag von G. Reimer, Berlin, 1882, 191-216.

Поступила в редакцию 20.11.2014, после доработки 24.02.2015 